\title{
A Consensus-Based Policy Framework on Pharmacist-Led Self-Management Using the Delphi Process
}

\author{
Fiona Y. Y. Wong ${ }^{1}$, Frank W. K. Chan ${ }^{1 *}$, Joyce H. S. You ${ }^{2}$, Eliza L. Y. Wong ${ }^{1}$, Eng-Kiong Yeoh ${ }^{1}$ \\ ${ }^{1}$ School of Public Health and Primary Care, The Chinese University of Hong Kong, Hong Kong, China; ${ }^{2}$ School of Pharmacy, The \\ Chinese University of Hong Kong, Hong Kong, China. \\ Email: fionawong@cuhk.edu.hk, ‘cwkfrank@cuhk.edu.hk, joyceyou@cuhk.edu.hk, lywong@cuhk.edu.hk, yeoh_ek@cuhk.edu.hk
}

Received June 25 ${ }^{\text {th }}$, 2012; revised July 26 ${ }^{\text {th }}$, 2012; accepted August $5^{\text {th }}, 2012$

\begin{abstract}
Fragmentation of the healthcare system and lack of connectivity between in-hospital and community services, have posed an impact on management of patients with chronic conditions. As self-management is an essential component in chronic disease management model and pharmacists are underutilized in Hong Kong, this study developed a consensus-based policy framework on pharmacist-led self-management using a Delphi panel consisted of medical doctors, pharmacists, Chinese medicine practitioners and dispensers. We had studied a local population survey to understand the use of over-the-counter medication, conducted focus groups and telephone survey to explore the views of the four health professions and the general population, respectively, on pharmacist-led approach in self-management. The findings served as the base for developing the self-administered questionnaire in Delphi. A total of 19 experts completed the questionnaires and rated the validity and clarity of each statement in a 9-point scale. The Delphi process was completed in two rounds. Community pharmacists could only play an assisting role in patient self-management. They should focus primarily on drug-related issues and were suggested to work on lifestyle modifications collaboratively with physicians and nurses. The government has to develop supportive measures to enhance pharmacist-led patient self-management in the future.
\end{abstract}

Keywords: Community Pharmacist; Self-Management; Consensus Policy Framework; Delphi Process

\section{Introduction}

The Hong Kong healthcare system is a dual system. It has a public sector and a private sector. Around $80 \%$ of outpatient care and primary care are provided largely by private sector and the bulk of specialist and inpatient secondary and tertiary care are delivered through the public sector. Because of the fragmentation of the current healthcare system and lack of connectivity between inhospital and community health services, many patients with chronic conditions are lack of support after receiving treatment from hospitals. Many patients are not able to manage their own health nor having healthcare professionals to monitor their chronic conditions before the next follow up, which generally takes three to six months. With the growing demand of health services as a result of aging population and advancement of medical technology, the response of the traditional model of health service delivery can no longer meet the needs. To fill the gap between in-hospital and community healthcare services in chronic disease, a new chronic care model needs to be considered.

\footnotetext{
${ }^{*}$ Corresponding author.
}

The Hospital Admission Risk Program (HARP) Chronic Disease Management Guidelines in Australia recommends healthcare professionals using a self-management approach in managing people with chronic diseases with or without complex needs [1]. In the Chronic Care Model, an organizing framework for improving chronic illnesses, self-management is also recognized as one of the elements [2]. It incorporates patient, provider and system level interventions with self-management support as a key component at the patient level. Previous studies have also supported that self-management programs can improve patients' self-efficacy, self-rated health, cognitive symptom management, exercise behaviors and communication with physicians [3-5].

Self-management in Hong Kong is still developing in an early stage. To choose a health professional qualifying to provide collaborative care to monitor patients' health conditions in self-management is another challenge. Physicians have the main role in disease diagnosis and management, and nurses also have the training and skills capable to monitor patients' conditions but it would be costly to have physicians to perform patient self-management and shortage of nursing manpower is an issue in the 
healthcare system. Community pharmacists in Hong Kong, however, are underutilized. Pharmacist is not the sole dispenser as medical doctors are also allowed to prescribe and dispense drugs. Patients usually receive prescribed medications from private doctors directly or from government hospital/clinic pharmacies after consultation. Pharmacists in hospitals may provide medication counseling to patients but other roles like disease monitoring, health promotion and education are uncommon. Studies have shown that pharmacists are the most accessible healthcare professional to many chronically ill patients [6] and given that they have limited roles, pharmacist can be considered to lead patient self-management.

To establish a policy in self-management of patients with chronic conditions using a pharmacist-led approach, a consensus-based policy framework involving medical doctors, pharmacists, traditional Chinese medicine (TCM) practitioners and dispensers needs to be developed first. Before conducting the Delphi process, we had studied the Thematic Household Survey to understand the use of western and Chinese over-the-counter medication in Hong Kong [7], conducted focus groups to explore the views of medical doctors, pharmacists, TCM practitioners and dispensers on patient self-management and role of pharmacist in self-management [8], and conducted a telephone survey to investigate the views of people over aged 18 on the pharmacist-led approach in self-management [9]. The findings of the focus group and telephone survey served as the base for developing the self-administered questionnaire in the Delphi process. TCM professionals are included in this study because it is a common practice to consult TCM practitioners in our community. Population studies have shown that $13 \%$ of $1,507,400$ persons who had consulted practitioners in the last 30 days, consulted TCM practitioners [10] and TCM practitioners have been recognized as a medical profession in Hong Kong since 1997.

The objective of this study was to develop a consensus-based policy framework on pharmacist-led selfmanagement using a Delphi panel consisted of medical doctors, pharmacists, TCM practitioners and dispensers.

\section{Materials and Methods}

\subsection{Subjects}

A total of 19 participants were invited for participation in the Delphi process. Diminishing returns suggest that Delphi respondent sample size of above 15 participants yields little improvement in reliability [11], therefore, we recruited around five participants from each of the professions. Five physicians, five pharmacists, five TCM practitioners and four TCM dispensers agreed to join. The recruitment was primarily through professional networks. Professional qualifications of participants and working experience were ensured. Inclusion on the panel required participants to meet the following criteria:

1) Current knowledge and understanding of patient selfmanagement and role of pharmacists;

2) Working in primary care and/or pharmacy sectors, including academic, clinical unit, community setting and/ or administration;

3) Commitment to contribute to each Delphi round.

\subsection{Instrument}

The self-administered Delphi questionnaires were written in both English and Chinese. In round one, the questionnaire composed of 53 statements on 1) patients involvement in self-management; 2) scope covered in patient self-management; 3) patients who may have difficulties in self-management; 4) roles of community pharmacists in self-management and; 5) barriers which may hinder pharmacist-led self-management. The statements were extracted from the focus group discussion [8], telephone survey [9] and expert opinions.

\subsection{Procedures}

Self-administered questionnaires were sent to the participants by post or email. In round one, participants were requested to rate the validity and clarity of each statement on a 9-point scale. Validity was defined as the extent to which the statement is relevant for the development of patient self-management or the role of community pharmacists in self-management of patients with chronic illness and; clarity was defined as the extent to which the statement was expressed in clear, precise and unambiguous language. A rating of $1-3$ was categorized as low validity/clarity; 4 - 6 as average validity/clarity and; 7 - 9 as high validity/clarity. Respondents were encouraged to provide feedback on how to improve validity and clarity of the statements, especially those with a rating of $1-6$. Statements were retained and needed not to be re-rated at round two if $85 \%$ or more of the respondents rated 7 - 9 on both validity and clarity, otherwise, the statements were revised at round one following the feedback of the participants or disregarded at round two.

In round two, each participant received a Delphi questionnaire showing his/her responses, the median and distribution of rating of each statement obtained in round one. Participants were asked to re-rate the validity and clarity of the revised statements on a 9-point scale. Same as in round one, a statement was retained if $85 \%$ or more of the respondents rated 7 - 9 on both validity and clarity, otherwise the statements were revised in the subsequent rounds or disregarded depending on the feedback of the participants.

\subsection{Data Analysis}

Descriptive statistics, including median and range of dis- 
tribution, were used to analyze responses in each round. Subgroup differences in rating between participants of western (physicians and pharmacists) and Chinese (TCM practitioners and dispensers) medicines were analyzed using SPSS (Version 16.0). Chi-square tests were performed to assess differences in ratings (7 - 9 vs 1 - 6) between the two subgroups. The level for statistical signifycance was set at $\mathrm{P}<0.05$.

\subsection{Ethical Consideration}

The study protocol was approved by the Joint CUHKNTEC Clinical Research Ethics Committee and was performed in accordance with the World Medical Association's Declaration of Helsinki.

\section{Results}

\subsection{Round One}

In the first round, consensus was achieved on 6 out of 53 statements and there was no significant difference in ratings (7 - 9 vs 1 - 6) between the western medicine and TCM groups. They included two statements on scope of self-management of patients with chronic illness (Medication compliance; Know when to seek help from healthcare providers); two statements on tasks of community pharmacists in pharmacist-led patient self-management (Handling drug problems and side-effects; Providing health product consultation); and two statements on barriers which may hinder community pharmacists to lead patient self-management (Patients' entire medical records and data are not available for community pharmacists; Liability issues for professional misconduct).

The remaining statements had less than $85 \%$ of participants rated them highly on validity and/or clarity. Twenty-one statements were disregarded as the participants commented that these statements were not valid/ relevant in the framework or some of them had similar meaning. Twenty-six statements were kept the same as in round one or revised for reassessment in round two.

\subsection{Round Two}

Among the 26 statements, three statements were disregarded because less than $85 \%$ rated validity and clarity 7 - 9. They were related to scope of self-management of patients with chronic illness (Checking of their own disease; and Aware of own treatment plan) and tasks of community pharmacists in pharmacist-led patient selfmanagement (Checking of patients' disease condition). Consensus was achieved on the remaining statements as more than $85 \%$ of respondents rated them highly on both validity and clarity. Besides, there was no significant difference on ratings between the western and Chinese medicine groups. The consensus-based policy framework on pharmacist-led self-management was, therefore, achieved after two rounds of Delphi process.

The following is a description of the consensus based policy framework (Table 1).

\subsection{Self-Management of Patients with Chronic Illness}

The four professions agreed that patients with stable chronic illnesses should be involved in self-management. Scope which should be covered in self-management included, medication and follow-up compliance, maintaining of healthy lifestyle, using proper channels to obtain disease information and know when to seek help from healthcare providers. However, the participants also identified numbers of barriers which might limit the ability of chronically ill patients in self-management. These barriers could basically be categorized into socio-demographic factors (old age and low education); mood and social cognition (pessimistic personality, emotional issues, and low perception of health, self-motivation and self-control); lack of disease and health knowledge (lack of health knowledge, not understand own disease, not following healthcare staff's instructions and incorrectly analyzing disease information) and poor health status.

\subsection{Role of Community Pharmacists in Patient Self-Management}

Community pharmacists could play an assisting role and they were unable to lead patient self-management. Handling drug related problems and providing health product consultation would be the major tasks of community pharmacists. Other tasks like health promotion, lifestyle modification with the collaboration of physicians and nurses, and referring patients to physicians were also included. The participants agreed that some barriers have to be tackled in order to advocate community pharmacists play a leading role in patient self-management in the future. The pharmacy profession lacked support from the government. Patients' medical records were not available for community pharmacists to perform effective selfmanagement. Some patients were not familiar with the roles of community pharmacists and the liability for professional misconduct was also a concern.

There were also some self-management activities and role of pharmacists, which were commonly found in patient self-management programs, but consensus were not achieved for these in this study. Other patient self-management activities which can be further considered include, monitoring of disease progress and complications, mental health and emotional support, and family/caregiver support. 
Table 1. The consensus based policy framework of self management of patients with chronic illness and role of pharmacists in patient self-management.

Part 1

Self management of patients with chronic illness

I. Patients with stable chronic illnesses should involve in self management.

II. Self management of patients with chronic illness should cover the following scope:

- Medication compliance

- Follow-up compliance

- Healthy lifestyle

- Able to use proper channel to obtain disease information

- Know when to seek help from health care providers

III. Chronic ill patients with the following characteristics or under the following circumstances, may have difficulties in patient self management:

- Old age

- Low education level

- Pessimistic personality

- Emotional issues

- Low perception of health

- Low self-motivation

- Low self-control (e.g. on diet control and medication compliance)

- Do not follow health care staff's instructions

- Lack of health knowledge

- Not understand their own disease

- Incorrectly interpreting disease information

- Unstable or poor prognosis of disease condition

Part 2

Role of community pharmacists in patient self management

I. Community pharmacists can play an assisting role in patient self management.

II. Community pharmacists can perform the following tasks in patient self management:

- Handling drug problems and side-effects

- Providing health product consultation

- Providing health promotion

- Working with physicians and nurses, assist patients in lifestyle modification

- With proper referral system and protocol, referring patients to physicians when necessary

III. The following barriers have to be minimized in order to have community pharmacists to lead patient self management in the future:

- Patients' entire medical records and data are not available for community pharmacists.

- Some patients are not familiar with the roles of community pharmacists.

- The community pharmacy profession lacks support from the government.

- Liability issues for professional misconduct 


\section{Discussion}

Generally, the four professions and the community population agreed that patients with chronic diseases should self manage their own illness provided that their health conditions were stable and they had sufficient knowledge on their diseases. Like many patients' self-management program conducted in other countries, the policy framework suggested that patients should obtain some basic disease information including symptoms, complications and treatments through appropriate channel, followed by drug and follow-up compliance and establishment of healthy lifestyle [12-16]. However, we should note that self-monitoring of disease parameters, such as blood pressure and blood glucose, awareness of own treatment plan, family and emotional support which were commonly covered in the self-management programs in other countries, were not included in our policy framework. This reflected that the concept and scope of patient selfmanagement identified in this study was quite different from the current practice.

The consensus based framework identified that patients with unstable and complicated conditions, emotional problems, who were very old, received low education, might not be able to self-manage their health. In Hong Kong, the education level of those aged 55 or older is generally low. According to census in 2006, 24\% had no schooling or received only pre-primary education while $30 \%$ completed high school [17]. Additionally, studies have found that people with low education usually receive low income and lack health knowledge [18-22]. Although the consensus based framework showed that these people might have difficulties in performing selfmanagement, the elderly and those who lack health knowledge should not be neglected in chronic disease management. Self-management programs have to be tailored to meet their special needs and health professionals/ educators need to have the skills and education materials developed to teach and communicate effectively with the disadvantaged group.

Though the training of pharmacists received in our region enable them to monitor patients' disease condition and parameters, provide health education and assist patients in lifestyle modification in addition to managing patients' drug issues, the consensus framework allowed pharmacists to play an assisting role only, primarily in drug management. Studies have been shown that physicians are most comfortable with pharmacists' responsibilities of detecting prescription errors, providing patient education, suggesting non-prescription medications and suggesting prescription medications to physicians [23]. They are willing to delegate tasks to pharmacists that they found difficult or mundane [24], but they are not giving up the authority to disease management and decision making in patient care. Our telephone survey inves- tigating the views of community population also showed similar results [9]. Approximately 45\% agreed pharmacists to lead patient self-management but $82 \%$ agreed on physician and pharmacist-led self-management. In Hong Kong, pharmacists working in hospitals and community believe that they have extended roles in the primary healthcare system [25]. They can be an educator on selfcare, an advisor to implement self-care, a specialist in follow-up of a patient's prescription, provision of drug counseling and formulating pharmaceutical care plans. However, their current roles are much limited in Hong Kong. Community pharmacists can be promoted to manage patients who were identified to be unable to perform self-management in the consensus framework, such as elderly, those with learning difficulties and unable to monitor own disease condition.

Based on the views of physicians, pharmacists, TCM practitioners and dispensers, without further work, it will not be possible to implement pharmacist-led patient selfmanagement. One of the reasons the other three professions did not agree community pharmacists to extend their roles in addition to drug management, was they lack interactions with community pharmacists and therefore they did not have confidence to extend the role of pharmacists. However, people lack interactions with community pharmacists because they have limited roles in healthcare services. To break the vicious cycle, in the reform to the healthcare delivery system, the government needs to consider the following:

\subsection{Strengthening the Multidisciplinary Approach in Primary Healthcare}

Traditionally, healthcare service and treatment are provided by individual professional discipline, however, studies have supported the effectiveness of multidisciplinary approach [26,27]. In Hong Kong, pharmacist-led patient self-management needs to be developed gradually with the support of government, including delivery of health services involving multidisciplinary team [28]. This would not only improve the quality of health services to patients but also ensure that our health professions are not underutilized. Both the health professionals and people in the community would have more exposure to other healthcare professionals rather than doctors and nurses alone.

\subsection{Right of Access of Pharmacists to an Electronic Health Record System}

Currently, the Hong Kong government is developing an electronic health record system which aims to connect all public and private hospitals. To facilitate pharmacist-led self-management, the right of access of pharmacists, especially the community pharmacists, to the system has 
to be considered. Lack of access to patients' medical record and medication information system is a primary obstacle for pharmacists to involve in patient self-management. If community pharmacists could access to the system, they could advise doctors on patients' prescription, monitor patients' disease condition more effectively, and facilitate provision of health education and suggestion on lifestyle modification to those in need. Physicians could in turn know the interventions that pharmacists had given to patients. The electronic health record system, therefore, not only improves effectiveness of case management, but also enables effective communication between professions.

\subsection{Advocating Continuing Pharmacy Education}

To ensure quality of care of pharmacists, continuing pharmacy education has to be advocated. In Hong Kong, there is no compulsory continuing professional education (CPE) for pharmacists. In our previous focus group study, physicians had revealed that they concerned the competency of pharmacists in handling tasks other than drug management [8]. CPE is important to pharmacists for updating, developing and acquiring new knowledge and skills and can enhance their career progression.

\subsection{Developing Equipment Subsidized Program}

Equipment subsidized by the government for screening and monitoring of patients' disease parameters in community pharmacies, and for patients to monitor their disease conditions at home are essential. Some small scale pharmacies are lack of adequate equipment to monitor patients' disease conditions. Similarly patients who have financial difficulties also may not be able to obtain the necessary equipment such as a blood pressure monitor or a glucometer, to perform self-management.

\subsection{Public-Private Partnership (PPP) with Community Pharmacists}

A PPP program can also be developed to encourage patients to consult community pharmacists for drug problems and to monitor drug compliance. By incorporating the PPP approach, the roles of community pharmacists can be promoted to the public, healthcare costs can be saved in long term and drug incidents can also be minimized. In the long run, the government may consider promotion of self-medication for minor ailments and the deregulation of more prescription only medicines to pharmacy only medicine as suggested by pharmacists in Hong Kong [29].

\section{Conclusion}

The consensus based policy framework indicated that community pharmacists could only assist in patient selfmanagement. The experts participated in the policy framework development agreed that pharmacists were drug experts, therefore, pharmacists will focus primarily on drug-related issues, including handling patients' drug problems and side-effects and providing drugs and health product consultation. Health promotion could be conducted by pharmacists alone but lifestyle modifications such as exercise planning, diet management and smoking cessation, pharmacists were suggested to work collaboratively with physicians and nurses. To enhance the implementation of pharmacist-led patient self-management in the primary healthcare system, supportive measures needed to be developed in order to extend the role of pharmacists gradually.

\section{Acknowledgements}

We thank all physicians, pharmacists, traditional Chinese medicine practitioners and dispensers who participated in the Delphi process, and the Studies in Health Services, Food and Health Bureau of the Hong Kong Special Administrative Region Government, financially supported the study.

\section{REFERENCES}

[1] Department of Human Services, "HARP Chronic Disease Management Guidelines,” Department of Human Services, Victoria, 2008.

[2] K. Fiandt, "The Chronic Care Model: Description and Application for Practice,” Advanced Practice Nursing, Vol. 6, No. 4, 2006.

[3] G. Foster, S. J. C. Taylor, S. Eldridge, J. Ramsay and C. J. Griffiths, "Self-Management Education Programmes by Lay Leaders for People with Chronic Conditions," Cochrane Database of Systematic Reviews, 2007. doi:10.1002/14651858.CD005108.pub2

[4] School of Medicine, "Chronic Disease Self-Management Program,” School of Medicine, Stanford. http://patienteducation.stanford.edu/programs/cdsmp.html

[5] A. M. H. Siu, C. C. H. Chan, P. K. K. Poon, D. Y. Chui, and S. C. Chan, "Evaluation of the Chronic Disease SelfManagement Program in a Chinese Population,” Patient Education and Counseling, Vol. 65, No. 1, 2007, pp. 4250. doi:10.1016/j.pec.2006.04.013

[6] The Pharmaceutical Society of Hong Kong \& the Society of Hospital Pharmacist of Hong Kong, "The Consultation Document on Health Care Reform 'Lifelong Investment in Health'," Hong Kong Special Administrative Region, Health and Welfare Bureau, 2011.

[7] V. C. H. Chung, C. H. Lau, F. W. K. Chan, J. H. S. You, E. L. Y. Wong, E. K. Yeoh, et al., "Use of Chinese and Western Over-the-Counter Medications in Hong Kong," Chinese Medicine, Vol. 5, 2010, p. 41. doi:10.1186/1749-8546-5-41

[8] F. Y. Y. Wong, F. W. K. Chan, J. H. S. You, E. L. Y. Wong 
and E. K. Yeoh, "Patient Self-Management and Pharmacist-Led Patient Self-Management in Hong Kong: A Focus Group Study from Different Healthcare Professionals' Perspectives,” BMC Health Services Research, Vol. 11, 2011, p. 121. doi:10.1186/1472-6963-11-121

[9] J. H. S. You, F. Y. Y. Wong, F. W. K. Chan, E. L. Y. Wong and E. K. Yeoh, "Self-Medication and Self-Care in Hong Kong,” BMC Clinical Pharmacology, Vol. 11, 2011, p. 19. doi:10.1186/1472-6904-11-19

[10] Census and Statistics Department, "Thematic Household Survey,” Report No. 30, Census and Statistics Department, Hong Kong Special Administrative Region, 2007.

[11] M. K. Murphy, N. A. Black, D. L. Lamping, C. M. McKee, C. F. Sanderson, J. Askham, et al., "Consensus Development Methods, and Their Use in Clinical Guideline Development," Health Technology Assessment, Vol. 2, No. 3, 1998, pp. 1-88.

[12] R. Holland, L. Brooksby, E. Lenaghan, K. Ashton, L. Hay, R. Smith, et al., "Effectiveness of Visits from Community Pharmacists for Patients with Heart Failure: Heart-Med Randomized Controlled Trial," British Medical Journal, Vol. 334, No. 7603, 2007, pp. 1098-2004. doi:10.1136/bmj.39164.568183.AE

[13] R. L. Rothman, R. Malone, B. Bryant, A. K. Shintani, B. Crigler, D. A. Dewalt, et al., “A Randomized Trial of a Primary Care-Based Disease Management Program to Improve Cardiovascular Risk Factors and Glycated Hemoglobin Levels in Patients with Diabetes," American Journal of Medicine, Vol. 118, No. 3, 2005, pp. 276-284. doi:10.1016/j.amjmed.2004.09.017

[14] D. Barbanel, S. Eldridge and C. Griffiths, "Can a Self-Management Programme Delivered by a Community Pharmacist Improve Asthma Control? A Randomised Trial," Thorax, Vol. 58, No. 10, 2003, pp. 851-854. doi:10.1136/thorax.58.10.851

[15] E. A. Coast-Senior, B. A. Kroner, C. L. Kelley and L. E. Trilli, "Management of Patients with Type 2 Diabetes by Pharmacists in Primary Care Clinics," The Annals of Pharmacotherapy, Vol. 32, 1998, pp. 636-641. doi:10.1345/aph.17095

[16] G. Foster, S. J. C. Taylor, S. Eldridge, J. Ramsay and C. J. Griffiths, "Self-Management Education Programmes by Lay Leaders for People with Chronic Conditions,” Cochrane Database of Systematic Reviews, 2007. doi:10.1002/14651858.CD005108.pub2

[17] Census and Statistics Department, "Thematic Report: Household Income Distribution in Hong Kong. 2006 Population By-Census,” Census and Statistics Department, Hong Kong Special Administrative Region, 2007.

[18] “Ontario Women Live Longer but Don't Prosper,” Daily Exchange, 2009. http://www.exchangemagazine.com/morningpost/2009/w eek26/Wednesday/062419.htm
[19] C. M. Deibert, S. Maliski, L. Kwan, A. Fink, S. E. Connor and M. S. Litwin, "Prostate Cancer Knowledge among Low Income Minority Men,” Journal of Urology, Vol. 177, No. 5, 2007, pp. 1851-1855. doi:10.1016/j.juro.2007.01.062

[20] Q. T. Zhang, D. Y. Hu, J. G. Yang, S. Y. Zhang, X. Q. Zhang and S. S. Liu, "Public Knowledge of Heart Attack Symptoms in Beijing Residents,” Chinese Medical Journal, Vol. 120, No. 18, 2007, pp. 1587-1591.

[21] P. Andersson and J. Leppert, "Men of Low-Socio-Economic and Educational Level Possess Pronounced Deficient Knowledge about the Risk Factors Related to Coronary Heart Disease,” Journal of Cardiovascular Risk, Vol. 8, No. 6, 2001, pp. 371-377. doi:10.1097/00043798-200112000-00006

[22] D. S. Kenkel, "Health Behavior, Health Knowledge, and Schooling,” Journal of Political Economy, Vol. 99, No. 2, 2001, pp.287-305. doi:10.1086/261751

[23] P. L. Ranelli and J. Biss, "Physicians’ Perceptions of Communication with and Responsibilities of Pharmacists," Journal of the American Pharmaceutical Association, Vol. 40, No. 5, 2000, pp. 625-630.

[24] J. Edmunds and M. W. Calnan, "The Reprofessionalisation of Community Pharmacy? An Exploration of Attitudes to Extend Roles for Community Pharmacists amongst Pharmacists and General Practitioners in the United Kingdom,” Social Science \& Medicine, Vol. 53, No. 7, 2001, pp. 943-955. doi:10.1016/S0277-9536(00)00393-2

[25] The Pharmaceutical Society of Hong Kong \& the Society of Hospital Pharmacist of Hong Kong, "The Consultation Document on Health Care Reform 'Lifelong Investment in Health',’ Hong Kong Special Administrative Region, Health and Welfare Bureau, 2001.

[26] J. A. Prvu Bettger and M. G. Stineman, "Effectiveness of Multidisciplinary Rehabilitation Services in Postacute Care: State-of-the-Science. A Review,” Archives of Physical Medicine and Rehabilitation, Vol. 88, No. 11, 2007, pp. 15261534. doi:10.1016/j.apmr.2007.06.768

[27] WHO Collaborating Centre for Hospitals and Health Promotion, "Feasibility, Effectiveness, Quality and Sustainability of Health Promoting Hospital Projects," Proceedings of the 5th International Conference on Health Promoting Hospitals, Vienna, 16-19 April 1997.

[28] F. Y. Wong, F. W. Chan, E. L. Wong and E. K. Yeoh, "Do Medical Students Agree with Pharmacist-Led Patient Self-Management in Hong Kong?” Medical Teacher, Vol. 32, No. 12, 2010. doi:10.3109/0142159X.2010.519076

[29] S. Pong, "Improving Hong Kong Health Care System: The Pharmacist Perspective. Topical Issues,” Legislative Council, Hong Kong Special Administrative Region, CB(2) 2521/98-99(01). http://www.legco.gov.hk/yr98-99/english/panels/hs/pape rs/2521e01.pdf 